\title{
Generators of lattice varieties
}

\author{
R. P. Dilworth* and Ralph Freese*
}

\section{Introduction}

Although it is well known that the variety of all lattices is generated by the subclass of finite lattices, there are lattice varieties which are not generated by their finite members. In fact, there are modular varieties which are not even generated by their finite dimensional members [3]. At the present time it is not known if the variety of all modular lattices is generated by its finite or even its finite dimensional members. This raises the question: Do there exist generators for lattice varieties which satisfy some kind of finiteness conditions? In this note we will be concerned with generators satisfying atomicity conditions. Since any lattice variety is generated by its subdirectly irreducible members, we shall be particularly interested in generators which are also subdirectly irreducible. The main results are the following. The notation and terminology for this paper is taken from [1].

THEOREM 1. Every lattice variety is generated by its strongly atomic members.

THEOREM 2. Every modular lattice variety is generated by its weakly atomic, subdirectly irreducible members.

THEOREM 3. Every modular lattice can be imbedded in a strongly atomic, subdirectly irreducible modular lattice.

It follows from Theorem 3, that the variety of all modular lattices is generated by its strongly atomic, subdirectly irreducible members. Whether a similar result holds for modular varieties in general is an open question.

Finally, we give some applications of Theorem 2.

Presented by B. Jónsson. Received April 14, 1975. Accepted for publication in final form September 9, 1975.

* This research supported in part by NSF Grants GP-35678 and GP-37772. 
Proofs of the Theorems. If $L$ is a lattice, the lattice of filters (dual ideals) of $L$ will be denoted by $\mathscr{F}(L)$. Principal filters will be identified with the corresponding elements of $L$, so that $L \subseteq \mathscr{F}(L)$. Inductively, we define $\mathscr{F}^{n}(L)=\mathscr{F}\left(\mathscr{F}^{n-1}(L)\right)$.

Proof of Theorem 1. For any lattice $L$ we have

$$
L \subseteq \mathscr{F}(L) \subseteq \cdots \subseteq \mathscr{F}^{n}(L) \subseteq \cdots
$$

We note that if $a>b$ in $\mathscr{F}^{n}(L)$, then $a>b$ in $\mathscr{F}^{n+1}(L)$. For if $(a)>C \geq(b)$ in $\mathscr{F}\left(\mathscr{F}^{n}(L)\right.$ then there exists $c \in C$ such that $c \geq a$. But then $a>a \cap c \geq b$ and hence $b=a \cap c$. Thus $b$ is contained in $C$ and hence $C=(b)$. Now let $L_{0}=\bigcup_{n} \mathscr{F}^{n}(L)$. Let $a, b \in L_{0}$ such that $a>b$. Then $a, b \in F^{n}(L)$ for some $n$ and hence there exists $c \in \mathscr{F}^{n+1}(L)$ such that $a \geq c>b$ in $\mathscr{F}^{n+1}(L)$. But then $c>b$ in $L_{0}$ and hence $L_{0}$ is strongly atomic. Finally, any identity satisfied by $L$ is satisfied by $\mathscr{F}(L)$ and hence by $F^{n}(L)$ for all $n$. But then $L_{0}$ satisfies every identity satisfied by $L$ and thus belongs to the variety generated by $L$. It follows that every variety is generated by its strongly atomic members.

Proof of Theorem 2. Let $L$ be a subdirectly irreducible modular lattice and let

$$
\mathscr{F}(L) \subseteq \prod_{\alpha} L_{\alpha}
$$

be a representation of $\mathscr{F}(L)$ as a subdirect product of subdirectly irreducible lattices $L_{\alpha}$. Let $\pi_{\alpha}$ denote the projection $\prod_{\alpha} L_{\alpha} \rightarrow L_{\alpha}$. Since $L$ is subdirectly irreducible, there exists elements $a, b \in L$ such that $a>b$ and $\theta a=\theta b$ for every nontrivial congruence relation $\theta$ on $L$. Let $a \geq c>b$ where $c \in \mathscr{F}(L)$. Then there exists a subscript $\alpha$ such that $\pi_{\alpha} c \neq \pi_{\alpha} b$. Since $\pi_{\alpha}$ is a surjection, it follows that $\pi_{\alpha} c>\pi_{\alpha} b$ in $L_{\alpha}$. By hypothesis $L_{\alpha}$ is subdirectly irreducible and modular. It follows that $\pi_{\alpha} c / \pi_{\alpha} b$ is weakly projective into every proper quotient of $L_{\alpha}$. Since $\pi_{\alpha} c / \pi_{\alpha} b$ is prime, every proper quotient of $L_{\alpha}$ contain a prime quotient and $L_{\alpha}$ is weakly atomic. Finally since $\pi_{\alpha} c \neq \pi_{\alpha} b$ we have $\pi_{\alpha} a \neq \pi_{\alpha} b$ and the mapping $x \rightarrow \pi_{\alpha} x$ is one-to-one on $L$. Thus $L$ is isomorphic to a sublattice of $L_{\alpha}$. Now every identity holding in $L$, also holds in $\mathscr{F}(L)$ and hence holds in $L_{\alpha}$. Clearly every identity holding in $L_{\alpha}$ also holds in every sublattice and hence holds in $L$. It follows that $L$ belongs to a variety if and only if $L_{\alpha}$ belongs to the variety and hence every modular variety is generated by its weakly atomic, subdirectly irreducible members.

Proof of Theorem 3. Let $L$ be a modular lattice and let $L_{0}=\mathscr{F}(L)$. Let $a_{\alpha} / b_{\alpha}$, $\alpha<\lambda$, be a well ordering of the prime quotients of $L_{0}$. For each $\alpha<\lambda$ we shall inductively construct a lattice $L_{\alpha}$ such that if $\beta<\alpha<\lambda, L_{\beta}$ is a sublattice of $L_{\alpha}$, 
coverings in $L_{\beta}$ are preserved in $L_{\alpha}$, and $a_{\alpha} / b_{\alpha}$ is projective to $a_{0} / b_{0}$ in $L_{\alpha}$. Let us suppose that $L_{\beta}$ has been constructed for $\beta<\alpha<\lambda$. Let $A=\mathscr{F}\left(\bigcup_{\beta<\alpha} L_{\beta}\right)$. By the dual of (6.2) of [1], we can find a completely join-irreducible element $a_{0}^{\prime}$ in $A$ such that $a_{0}^{\prime} \leq a_{0}$ and $a_{0}^{\prime} \leq b_{0}$. If we set $b_{0}^{\prime}=b_{0} \wedge a_{0}^{\prime}$, then $a_{0} / b_{0}$ and $a_{0}^{\prime} / b_{0}^{\prime}$ are projective.

We will find it convenient to make repeated use of the following construction from [5]. Let $X$ and $Y$ be modular lattices such that a principal ideal $Z$ of $X$ is isomorphic to a principal dual ideal $Z^{\prime}$ of $Y$. Then the set $X \cup Y$ with $Z$ and $Z^{\prime}$ identified can be made into a modular lattice by defining $a \leq b$ if and only if $a \leq b$ in $X$ or $a \leq b$ in $Y$ or if $a \leq z^{\prime}, z \leq b$ where $z$ and $z^{\prime}$ are corresponding elements under the isomorphism of $Z$ to $Z^{\prime}$.

Let $B=\left\{a \in A: a \leq b_{0}^{\prime}\right\}$. Let $x, y, z$ be the atoms of a copy of $M_{5}$ with unit and null elements $u$ and $v$ respectively. Applying the above construction to $B \times \mathbf{2}$ and $M_{5}$ where $\left(b_{0}^{\prime}, 1\right) /\left(b_{0}^{\prime}, 0\right)$ is identified with $x / v$ we get a modular lattice $C$. Since $a_{0}^{\prime}$ is completely join irreducible in $A$, the ideal $a_{0}^{\prime} / 0$ in $A$ is isomorphic to the dual ideal $u /(0,1)$ of $C$. Identifying isomorphic elements and using the above construction gives a lattice $D$. If we let $a_{0}^{\prime \prime}=(0,1)$ and $b_{0}^{\prime \prime}=(0,0)$, then in $D$ we have

$$
a_{0} / b_{0}^{\prime} \searrow a_{0}^{\prime} / b_{0}^{\prime} \searrow y /\left(b_{0}^{\prime}, 0\right) \nearrow u / z \searrow x / v \searrow a_{0}^{\prime \prime} / b_{0}^{\prime \prime}
$$

Note that $A$ is isomorphic to the principal dual ideal of $D$ generated by $(0,1)$.

We now repeat the entire process starting with $a_{\alpha} / b_{\alpha}$ in $D$ rather than $a_{0} / b_{0}$ in $A$. In the resulting lattice $E, a_{\alpha} / b_{\alpha}$ is projective to $(0,1) /(0,0)$. Thus in $E$ there are two distinct atoms $c_{0}$ and $c_{\alpha}$ such that $a_{0} / b_{0}$ is projective to $c_{0} / 0$ and $a_{\alpha} / b_{\alpha}$ is projective to $c_{\alpha} / 0$.

Let $x_{i}, y_{i}, z_{i}$ for $i=1,2,3$ be the atoms of three copies of $M_{5}$ with null element $v_{i}$ and unit element $u_{i}, i=1,2,3$. Let $F$ be the lattice obtained from the first two copies of $M_{5}$ by identifying $x_{2}$ with $v_{1}$ and $u_{2}$ with $z_{1}$. Let $G$ be the lattice obtained from the union of $E$ and the third copy of $M_{5}$ by identifying $c_{0}$ with $u_{3}$ and 0 with $x_{3}$. Finally let $L_{\alpha}$ be the lattice obtained from $F \cup G$ by identifying $u_{1}$ with $c_{\alpha}, u_{2}=z_{2}$ with $0=x_{3}$, and $z_{1}$ with $v_{3}$. Clearly $L_{\beta}$ is isomorphic to a sublattice of $L_{\alpha}$ for all $\beta<\alpha$. Since coverings are preserved in the natural imbedding in the dual ideal lattice, coverings in $L_{\beta}$ are preserved in $L_{\alpha}$. Finally since $c_{0} / 0$ and $c_{\alpha} / 0$ in $E$ are projective in $L_{\alpha}$ to prime quotients of $F$, it follows that $a_{\alpha} / b_{\alpha}$ and $a_{0} / b_{0}$ are projective in $L_{\alpha}$.

Let $M_{1}=\bigcup_{\alpha<\lambda} L_{\alpha}$. We repeat the entire procedure with $M_{1}$ in place of $L$. In this manner we obtain $M_{2}$ and inductively $M_{2}$ and inductively $M_{3}, M_{4}, \ldots$ Let $M=\bigcup M_{i}$. Then, as in the proof of Theorem $1, M$ is strongly atomic. Since all prime quotients of $M$ are projective, $M$ is subdirectly irreducible. This completes the proof of Theorem 3 . 
Although the techniques of the proof of Theorem 3 do not apply to all varieties of modular lattices, there are certain lattice identities which are preserved under this construction. The most important of these is the Arguesian law.

\section{Application}

The study of varieties of modular lattices is often facilitated if the variety is generated by subdirectly irreducible lattices containing prime quotients. Theorem 2 shows that we may always assume this to be the case. For example; the proofs of the results in [2] concerning modular varieties can be considerably shortened with the aid of Theorem 2 (see Appendix B of [2]). On the other hand, it should be pointed out that the full structural results of [2] cannot be obtained in this way. Theorem 2 may also be used to shorten the proofs of some of the results of [6].

As another application of Theorem 2, consider the result of B. Jónsson [7] which states that a modular lattice $L$ not in the variety generated by $M_{5}$ has a homomorphic image of a sublattice isomorphic to one of the two lattices of Figure 1.
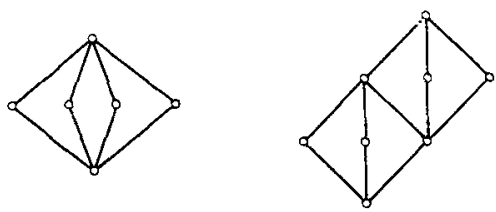

Figure 1

G. Grätzer [4] obtained this result under the assumption that $L$ is finite. It follows from the more general result of Jonnson that the variety. generated by $M_{5}$ is strongly covered by two modular varieties. Since the Grätzer proof depends only on $L$ having a prime quotient, the covering property for the variety generated by $M_{5}$ follows from Grätzer's result and Theorem 2. Again, it should be noted that the full structural result of Jónsson cannot be recovered in this manner.

\section{REFERENCES}

[1] P. Crawley and R.P. Dilworth, Algebraic Theory of Lattices, Prentice-Hall, Englewood Cliffs, N.J. 1973.

[2] R. FREESE, The structure of modular lattices of width four with applications to varieties of lattices, Memoir. Amer. Math. Soc. (to appear).

[3] - Some varieties of modular lattices not generated by their finite dimensional members, Proc. of the Szeged Universal Algebra Conference. 
[4] G. GRÄTZER, Equational classes of lattices, Duke Math. J. 33 (1966), 613-622.

[5] M. HALL and R. P. Dilworth, The embedding problem for modular lattices, Annals of Math. 45 (1944), 450-456.

[6] D. X. Hong, Covering relations among lattice varieties, Pacific J. Math. 40 (1972), 575-603.

[7] B. Jónsson, Equational classes of lattices, Math. Scand. 22 (1968), 187-196.

California Institute of Technology Pasadena, California

U.S.A.

University of Hawaii

Honoululu, Hawaii

U.S.A. 